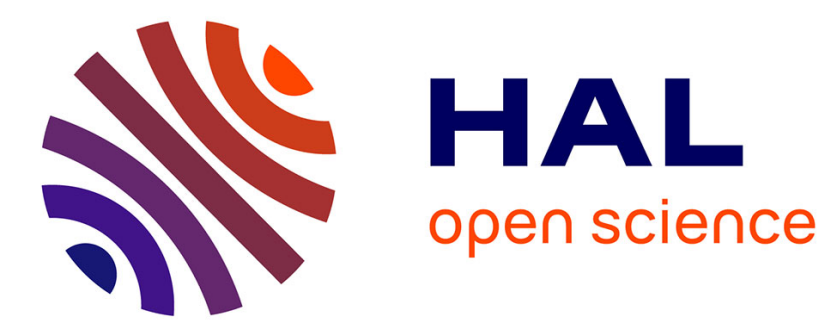

\title{
Double grid diffuse collocation method
}

Piotr Breitkopf, Gilbert Touzot, Pierre Villon

\section{To cite this version:}

Piotr Breitkopf, Gilbert Touzot, Pierre Villon. Double grid diffuse collocation method. Computational Mechanics, 2000, 25 (2-3), pp.199-206. 10.1007/s004660050469 . hal-01994275

\section{HAL Id: hal-01994275 \\ https://hal.utc.fr/hal-01994275}

Submitted on 19 Aug 2019

HAL is a multi-disciplinary open access archive for the deposit and dissemination of scientific research documents, whether they are published or not. The documents may come from teaching and research institutions in France or abroad, or from public or private research centers.
L'archive ouverte pluridisciplinaire HAL, est destinée au dépôt et à la diffusion de documents scientifiques de niveau recherche, publiés ou non, émanant des établissements d'enseignement et de recherche français ou étrangers, des laboratoires publics ou privés. 


\title{
Double grid diffuse collocation method
}

\author{
P. Breitkopf, G. Touzot, P. Villon
}

\begin{abstract}
In the present paper we propose a new method for constructing a second order Moving Least Squares (MLS) approximation. The method leads to shape functions which are then used for solving Partial Differential Equations (PDE) by a collocation method. This work is an extension of the Generalized Finite Difference Method originally proposed by Liszka and Orkisz (GFDM). However it differs from GFDM by using a sequence of two first order numerical derivations based on linear polynomial basis instead of a second order derivation based on a quadratic polynomial basis. This two-stage approach leads to continuous approximation coefficients using a limited number of surrounding points and results into quite a simple program structure, very similar to that of the finite elements. The method is in an early stage of development so no definitive conclusions may be drawn, however example problems exhibit good convergence properties.
\end{abstract}

\section{1}

\section{Introduction}

The meshless collocation approach was originally introduced by Liszka et al. (1980) under the denomination "Generalized Finite Difference Method" (GFDM). The equilibrium equations of continuum involving second order derivatives of displacements, their evaluation in two dimensions based on nodal displacements, requires a quadratic polynomial basis. Thus, the evaluation of approximation coefficients demands a connectivity of at least 6 nodes. The choice of these supporting nodes is constrained as particular patterns lead to degenerated solutions [Syczewski (1981)]. The selection follows a two steps approach. First, at an evaluation point $\mathbf{x}$, a set of "closest nodes" is selected inside a radius of influence $r(\mathbf{x})$. In the second step, several conditions [Sect. 3.4 in Liszka et al. (1996)] are applied in order to ensure sufficient "star quality" by selecting a subset of nodes among those belonging to $r(\mathbf{x})$ vicinity. This approach presents two drawbacks:

- the domains of definition of the shape functions are complex and correspond to Voronoï diagrams of an

P. Breitkopf

Université de Technologie de Compiègne, BP 20529, F-60205

Compiègne, Cedex, France

G. Touzot

INSA de Rouen, Rouen, France

P. Villon ( $\square)$

Université de Technologie de Compiègne, BP 20529, F-60205

Compiègne, Cedex, France order depending on the number $n$ of points taken into account, $n \geq 6$.

- the selection of nodes spoils the continuity of the Moving Least Squares approximation.

In the present work we use a different strategy: the radius of influence $r(i)$ is not evaluated at the evaluation point, but it is bounded to the node $i$. Thus the domains of definition of the shape functions obtain straightforward shapes (circular or rectangular depending on the norm chosen for the computation of the distance between the evaluation point and the nodes). In order to fully benefit from the MLS properties one has to take into account all the nodes containing the evaluation point in their domain of influence. On the other hand, the number of nodes should be limited in order to keep the global matrix sparse. In this work we propose a compromise by splitting the second order differential operator into two parts requiring limited nodal support thus permitting to reduce the size of the domains of influence.

The main guideline of the present work is to replace the second order differentiation scheme by a sequence of two first order schemes. We introduce two sets of discretization points: nodes and evaluation points. Their roles are respectively similar to that of nodes and of integration points in finite elements. Discrete values of the unknown functions (displacements) are attached to the nodes. In a first step the gradients (strains) of the unknown functions are computed at the evaluation points by a first order discrete differential operator applied to nodal unknowns. Then, like in the finite element method, the constitutive relations are written at the evaluation points, leading to stresses. In the second step the classical integration of the stiffness matrix over each individual finite element, is replaced by the direct evaluation of the equilibrium equations at the nodes, using a first order differential operator applied to stresses. Both derivation operators being first order ones, they only require a limited connectivity. The strains are continuous functions of the coordinates of any point $\mathbf{x}$ if 4 or more nodes are connected to that point. In a similar way a continuous evaluation of the equilibrium equations at any point $\mathbf{x}$ only requires 4 evaluation points to be connected to that point. In practical approaches these numbers may be higher than 4 but the connectivity is significantly smaller than when using a simple grid technique.

\section{2}

Double grid diffuse approximation and derivation

The governing idea of the "diffuse" [Nayroles et al. (1992)] and "meshless" [Belytschko et al. (1994)] methods consists 


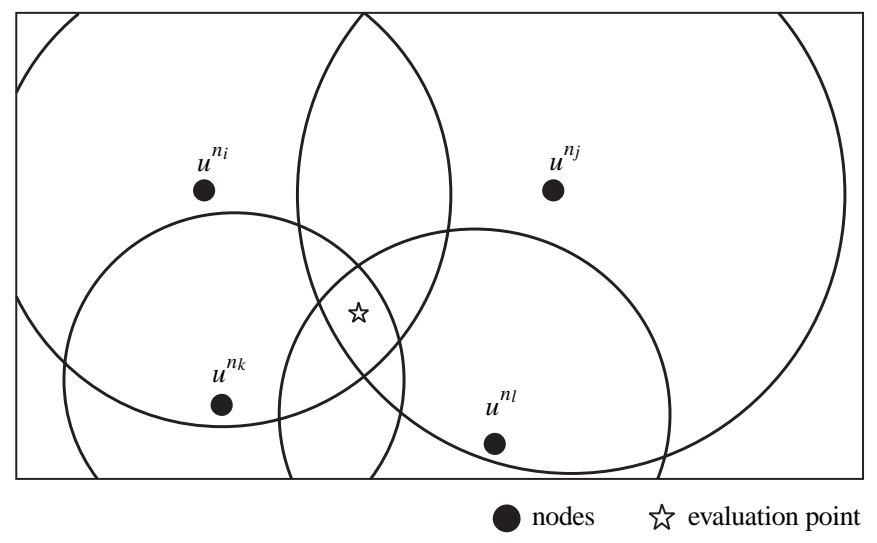

Fig. 1. An evaluation point located in the intersection of the domains of influence of a set of four nodes

in solving partial differential equations (PDE) without using explicit finite element meshes. An unknown function is represented by its discrete values at a set of nodes. The influence of the value of the function at a nodes $\mathbf{x}_{i}$ on the value at an evaluation point $\mathbf{x}$ close to $\mathbf{x}_{i}$ is weighted by some weight function $w_{i}\left(\left\|\mathbf{x}-\mathbf{x}_{i}\right\|\right)$. This function vanishes outside a domain of influence of the node $\mathbf{x}_{i}$.

In the present paper, we focus on collocation methods. The discrete equilibrium equations of continuous media generally involve second order differential operator $L$ applied to an unknown function $u(\mathbf{x})$ in the interior of the domain. First order operator $P$ also appears, in particular for evaluation of stresses and Neumann-type boundary conditions. Let us consider the approximation of second order derivatives using a two steps and double grid approach.

First, we start from a given set of nodal function values $u\left(\mathbf{x}_{j}^{n}\right)$ at an irregular grid of nodes $\mathbf{x}_{j}^{n}, j=1, \ldots, n_{n}$. We approach the discrete gradients $P u(\mathbf{x})$ of the function at an arbitrary set of evaluation points $\mathbf{x}_{i}^{P E}, i=1, \ldots, n_{P E}$ by a linear combination of the nodal function values $u\left(\mathbf{x}_{j}^{n}\right)$ whose coefficients are noted $N_{j, P}\left(\mathbf{x}_{i}^{P E}\right)$

$u_{, P}\left(\mathbf{x}_{i}^{P E}\right)=P u\left(\mathbf{x}_{i}^{P E}\right)=\sum_{j} N_{j, P}\left(\mathbf{x}_{i}^{P E}\right) u\left(\mathbf{x}_{j}^{n}\right)$

Next, we approach the second derivatives $L u(\mathbf{x})$ of the function at a point $\mathbf{x}$ by a linear combination of $u_{, P}\left(\mathbf{x}_{i}^{P E}\right)=P u\left(\mathbf{x}_{i}^{P E}\right)$ whose coefficients are noted $M_{i, L}(\mathbf{x})$

$L u(\mathbf{x})=\sum_{i} M_{i, L}(\mathbf{x}) u_{, P}\left(\mathbf{x}_{i}^{P E}\right)$

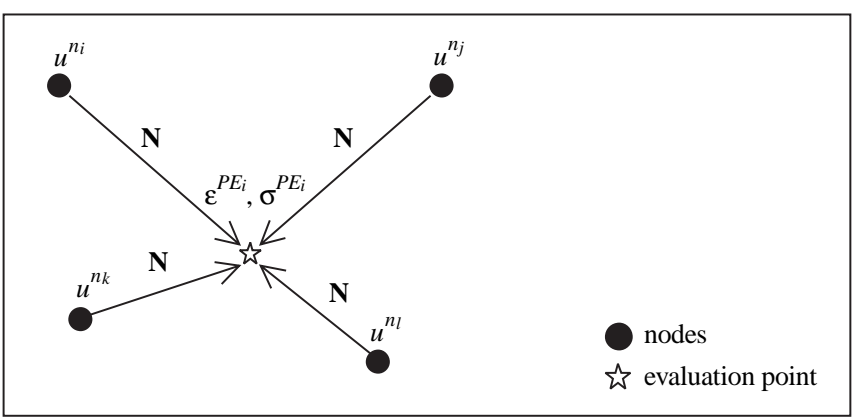

Fig. 2. First step: strains and stresses at an evaluation point computed from the nodal displacements at nodes
Figure 2 illustrates the first step in the framework of continuum mechanics. Displacements are known at nodes and the strains are approximated at the evaluation points.

Figure 3 illustrates the second step. The equilibrium equations are evaluated at the nodes from the stresses approximated at the evaluation points.

The combination of (1) and (2) gives

$$
\begin{aligned}
L u(\mathbf{x}) & =\sum_{j} \sum_{i} M_{i, L}(\mathbf{x}) N_{j, P}\left(\mathbf{x}_{i}^{P E}\right) u\left(\mathbf{x}_{j}^{n}\right)=\sum_{j} \alpha_{j} u\left(\mathbf{x}_{j}^{n}\right) \\
\alpha_{j} & =\sum_{i} M_{i, L}(\mathbf{x}) N_{j, P}\left(\mathbf{x}_{i}^{P E}\right)
\end{aligned}
$$

We shall now explicit the conditions that have to be satisfied by the coefficients $\alpha_{j}$ in order to guarantee the convergence of $L u(\mathbf{x})$. Substituting a Taylor series expansion of $u\left(\mathbf{x}_{j}^{n}\right)$ centered at $\mathbf{x}$ into (3) we get

$$
\begin{aligned}
L u(\mathbf{x})= & \sum_{j} \alpha_{j} u(\mathbf{x})+\sum_{j} \alpha_{j}\left(x_{j}^{n}-x\right) u_{, x}(\mathbf{x}) \\
& +\sum_{j} \alpha_{j}\left(y_{j}^{n}-y\right) u_{, y}(\mathbf{x})+\frac{1}{2} \sum_{j} \alpha_{j}\left(x_{j}^{n}-x\right)^{2} u_{, x x}(\mathbf{x}) \\
& +\frac{1}{2} \sum_{j} \alpha_{j}\left(y_{j}^{n}-y\right)^{2} u_{, y y}(\mathbf{x}) \\
& +\sum_{j} \alpha_{j}\left(x_{j}^{n}-x\right)\left(y_{j}^{n}-y\right) u_{, x y}(\mathbf{x})+\cdots
\end{aligned}
$$

It may be conveniently written as

$L u(\mathbf{x}) \cong(\mathbf{P a})^{\mathrm{T}}\left\{\begin{array}{c}u \\ u_{, x} \\ u_{, y} \\ u_{, x x} \\ u_{, y y} \\ u_{, x y}\end{array}\right\}$

where $\mathbf{P}$ is the matrix of consistency constraints (not to be confused with the differential operator $P$ from Eq. (1) and has the following form

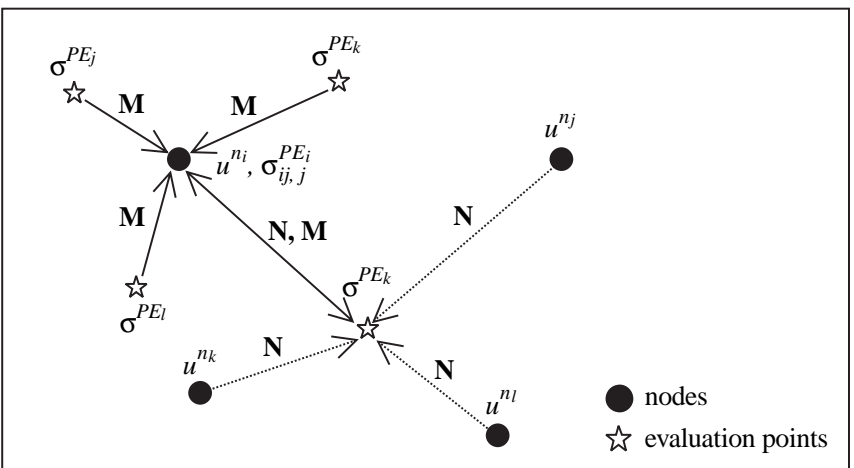

Fig. 3. Second step: equilibrium equations are obtained at the nodes from the stress values known at the evaluation points 
$\mathbf{P}=\left[\begin{array}{c}1 \\ x_{j}^{n}-x \\ y_{j}^{n}-y \\ \ldots \\ \frac{1}{2}\left(x_{j}^{n}-x\right)^{2} \\ \frac{1}{2}\left(y_{j}^{n}-y\right)^{2} \\ \left(x_{j}^{n}-x\right)\left(y_{j}^{n}-y\right)\end{array}\right], \quad \mathbf{a}=\left\{\begin{array}{c}\vdots \\ \alpha_{j} \\ \vdots\end{array}\right\}$

When the conditions

$\mathbf{P a}=\mathbf{e}^{i}$

with

$\mathbf{e}^{i}=\left\{\begin{array}{c}\vdots \\ \delta_{i j} \\ \vdots\end{array}\right\}, \quad \begin{cases}\delta_{i j}=1 & \text { when } i=j \\ \delta_{i j}=0 & \text { when } i \neq j\end{cases}$

are satisfied for $i=1,2,3,4,5,6$ then the operator $L$ defined by (4) converges respectively towards

$L \equiv I, \quad L \equiv \frac{\partial}{\partial x}, \quad L \equiv \frac{\partial}{\partial y}, \quad L \equiv \frac{\partial^{2}}{\partial x^{2}}$,

$L \equiv \frac{\partial^{2}}{\partial y^{2}}, \quad L \equiv \frac{\partial^{2}}{\partial x^{2}}, \quad L \equiv \frac{\partial^{2}}{\partial x \partial y}$

Conditions (5) may be rewritten as two independent sets of constraints acting respectively on vectors $\mathbf{N}$ and $\mathbf{M}$ collecting coefficients $N_{i}$ and $M_{i}$ introduced in (1) and (2)

$\mathbf{Q N}=\mathbf{e}^{k}$

and

$\mathbf{R M}=\mathbf{e}^{l}$

Developing (3) we see that an interesting choice for the matrix $\mathbf{Q}$ is

$\mathbf{Q}=\left[\begin{array}{ccc} & 1 & \\ \cdots & x_{j}^{n}-x & \cdots \\ & y_{j}^{n}-y & \end{array}\right]$

The matrix $\mathbf{R}$ becomes in this case

$\mathbf{R}=\left[\begin{array}{ccc} & \begin{array}{c}1 \\ \frac{1}{2} \sum_{j=1, n_{P E}} N_{j, P}\left(x_{j}^{P E}-x\right)^{2}\end{array} \\ \cdots & \frac{1}{2} \sum_{j=1, n_{P E}} N_{j, P}\left(y_{j}^{P E}-y\right)^{2} & \cdots \\ & \sum_{j=1, n_{P E}} N_{j, P}\left(x_{j}^{P E}-x\right)\left(y_{j}^{P E}-y\right)\end{array}\right]$

As the number of data points (nodes) taken into account is generally greater than the number of constraints, the coefficients $\mathbf{N}$ may be obtained by minimizing the following criteria

$\min \left(\mathbf{N}^{\mathrm{T}} \mathbf{W}_{n}^{-1} \mathbf{N}\right)$

where $\mathbf{W}_{n}$ is a diagonal matrix containing nodal weight function values $w_{i}\left(\left\|\mathbf{x}_{i}^{P E}-\mathbf{x}_{j}^{n}\right\|\right)$. The solution of (10) under the constraints (6) may be obtained from the associated Lagrangian (Breitkopf et al. (1998)) and gives

$\mathbf{N}^{\mathrm{T}}=\mathbf{e}^{k \mathrm{~T}}\left(\mathbf{Q} \mathbf{W}_{n} \mathbf{Q}^{\mathrm{T}}\right)^{-1} \mathbf{Q} \mathbf{W}_{n}$ for different choices of $k$.

The particular case $k=1$ produces the vector $\mathbf{N}$ corresponding to the shape functions of the first order Moving Least Squares (MLS) approximation while $k=2$ and $k=3$ yield the MLS diffuse derivatives.

The coefficients $\mathbf{M}$ may be obtained by minimizing

$\min \left(\mathbf{M}^{\mathrm{T}} \mathbf{W}_{P E}^{-1} \mathbf{M}\right)$

under the constraints (7) where $\mathbf{W}_{P E}$ is a diagonal matrix of weights $w_{i}\left(\left\|\mathbf{x}-\mathbf{x}_{i}^{P E}\right\|\right)$

$\mathbf{M}^{\mathrm{T}}=\mathbf{e}^{l^{\mathrm{T}}}\left(\mathbf{R W}_{P E} \mathbf{R}^{\mathrm{T}}\right)^{-1} \mathbf{R} \mathbf{W}_{P E}$

For the particular case of $k=2$ the following relations are readily obtained for different choices of $l$ :

- $l=1$ leads to a second order approximation of the $x$ derivative at the node,

- $l=2$ gives approximation coefficients for the second derivative,

$-l=4$ leads to an approximation of the second order mixed derivative.

The constraint corresponding to $k=3$ may be combined with:

- $l=1$ giving a second order approximation of the $y$ derivative at the node,

- $l=3$ leads to approximation coefficients for the second derivative with respect to $y$,

- $l=4$ gives a different approximation of the mixed derivative.

These results are summarized in the Table 1. One may note, that the approximation of the mixed derivative $u_{x y}$ may be obtained in two different ways, either by choosing $k=2$ and $l=4$ or $k=3$ and $l=4$. The corresponding numerical values are slightly different.

As mentioned, the choice (8), (9) is not unique and a particular case consists in taking the matrix $\mathbf{Q}$ equal to $\mathbf{P}$

$\mathbf{Q} \equiv \mathbf{P}$

in this case the relation (7) reduces to a partition of unity

$\mathbf{R}=\left[\begin{array}{llll}1 & 1 & \cdots & 1\end{array}\right]$

and the double grid is equivalent to second order MLS used in the GFDM method.

Although the convergence properties of the double grid are the same as those of second order MLS, the two-steps approach is interesting for several reasons:

- The continuity of the one-step second order MLS (12), (13) requires the domains of influence of the nodes to be defined in such a way to ensure an intersection

Table 1. Shape functions obtained from (10) under constraints (6) for different values of $k$ and from (11) under constraints (7) for different choices of $l$

\begin{tabular}{lllll}
\hline & $l=1$ & $l=2$ & $l=3$ & $l=4$ \\
\hline$k=1$ & $N, M$ & & & \\
$k=2$ & $N_{, x}, M_{, x}$ & $N_{, x}, M_{, x x}$ & & $N_{, x}, M_{, x y}$ \\
$k=3$ & $N_{, y}, M_{, y}$ & & $N_{, y}, M_{, y y}$ & $N_{, y}, M_{, y x}$ \\
\hline
\end{tabular}




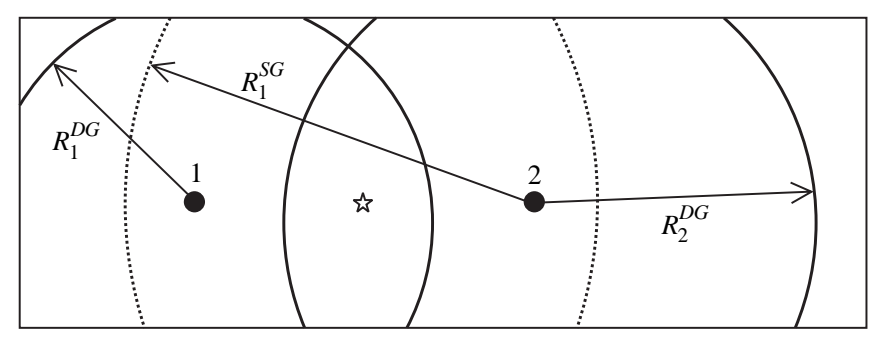

nodes

is intermediary point

Fig. 4. Coupling of nodes through an evaluation point requires smaller domains of influence (solid lines, $\mathbf{R}^{D G}$ ) than a direct coupling of nodes (dotted lines, $\mathbf{R}^{S G}$ )

of more than 6 domains at each evaluation point. In the case of highly irregular grids this requirement leads to big domains of influence. At an evaluation point this results either in an explosive connectivities $n \gg 6$ or we have to select a subset (node stencils in Liszka et al. (1996)) of surrounding nodes but in the latter case we give up the continuity of the approximation.

- DG requires a limited connectivity to be continuous: more than 3 nodes for the first grid and at least 4 points for the second grid.

- In practical application the choice of the primary grid of nodes may be imposed for instance by CAD. Evaluation points may be generated in order to obtain better conditioning of the overall system. This aspect is thoroughly discussed by Breitkopf et al. (1998).

- The points where the constitutive relations are evaluated are different from the points where we are looking for the nodal unknowns (displacements, velocities,...). This feature may be useful in particular cases like multiphase or layered media.

\section{3}

\section{Double grid diffuse collocation method}

Let us consider a few examples of application of the double grid approximation. We begin with Poisson's equation

$$
\begin{aligned}
k_{x} \frac{\partial^{2} u}{\partial x^{2}}+k_{y} \frac{\partial^{2} u}{\partial y^{2}} & =f(x, y), \\
& (x, y) \in \Omega, \quad \Omega=(-1,1) *(-1,1)
\end{aligned}
$$

with essential (Dirichlet) and natural (Neumann) boundary conditions

$$
\begin{aligned}
& u=\hat{u}, \quad(x, y) \in \partial \Omega_{D} \\
& \frac{\partial u}{\partial x} n_{x}+\frac{\partial u}{\partial y} n_{y}=g(x, y), \quad(x, y) \in \partial \Omega_{N}
\end{aligned}
$$

We shall use the first grid in order to represent the derivatives $\frac{\partial u}{\partial x}\left(\mathbf{x}^{P E}\right)$ and $\frac{\partial u}{\partial y}\left(\mathbf{x}^{P E}\right)$ based on the nodal values $u\left(\mathbf{x}^{n}\right)$

$$
\left\{\begin{array}{c}
\frac{\partial u}{\partial x}\left(\mathbf{x}^{P E}\right) \\
\frac{\partial u}{\partial y}\left(\mathbf{x}^{P E}\right)
\end{array}\right\}=\mathbf{B}\left\{\begin{array}{c}
\vdots \\
u_{i} \\
\vdots
\end{array}\right\}
$$

the $\mathbf{B}$ matrix being analogous to that of the finite element method

$\mathbf{B}=\left[\begin{array}{lll}\cdots & N_{, x} & \cdots \\ \cdots & N_{, y} & \cdots\end{array}\right]$

The Laplace's operator may be written at a node as a linear combination of the gradient values at surrounding evaluation points with coefficients $M_{, x x}$ and $M_{, y y}$

$$
\begin{aligned}
& k_{x} \frac{\partial^{2} u}{\partial x^{2}}\left(\mathbf{x}^{n}\right)+k_{y} \frac{\partial^{2} u}{\partial y^{2}}\left(\mathbf{x}^{n}\right) \\
& \quad=\sum_{i}\left[k_{x} M_{i, x x}\left(x^{n}\right) k_{y} M_{i, y y}\left(x^{n}\right)\right]\left\{\begin{array}{l}
\frac{\partial u}{\partial x}\left(\mathbf{x}_{i}^{P E}\right) \\
\frac{\partial u}{\partial y}\left(\mathbf{x}_{i}^{P E}\right)
\end{array}\right\}
\end{aligned}
$$

This operation performed for all nodes leads to a discretization of (14) and it may be performed in two loops

- the first loop over the evaluation points in order calculate the matrices $\mathbf{B}$

- the second loop over the nodes writing one discrete equation for each node.

The operations involved in the second loop may be reordered in such a way to be performed over the evaluation points. Then the whole process results into a single loop over evaluation points instead of two independent loops. In this modified scheme the equations are progressively built, by assembling the contributions of each individual evaluation point to the equations bounded to all the nodes located in its vicinity. The corresponding contribution of one evaluation point is

$\mathbf{B T} \cdot \mathbf{D} \cdot \mathbf{B}\left\{\begin{array}{c}\vdots \\ u_{i} \\ \vdots\end{array}\right\}=\mathbf{k}^{P E}\left\{\begin{array}{c}\vdots \\ u_{i} \\ \vdots\end{array}\right\}, \quad \mathbf{D}=\left[\begin{array}{cc}k_{x} & 0 \\ 0 & k_{y}\end{array}\right]$

where $\mathbf{D}$ is the constitutive matrix and BT is given by

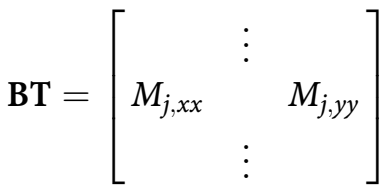

and the assembly of the global system may be performed in the finite element like manner

$$
\left.\sum_{\substack{\text { evaluation } \\ \text { points }}} \mathbf{k}_{i}^{P E}\right) \mathbf{u}=\mathbf{K u}=\mathbf{f}
$$

The BT matrix plays a role similar to the $\mathbf{B}^{\mathrm{T}}$ matrix used in finite elements: it introduces the notion of elementary matrix $\mathbf{k}_{\mathrm{e}}$ bound to an evaluation point. The difference with finite elements is that no numerical integration is performed. $\mathbf{B T} \neq \mathbf{B}^{\mathrm{T}}$ and the elementary matrix $\mathbf{k}_{\mathrm{e}}$ are not symmetric. There is a certain analogy between the evaluation point in the double grid method and an integration (Gauss) point in the finite element method. An evaluation point producing an elementary matrix may be viewed as a finite element on its own. Such a "diffuse collocation 
element" may be easily inserted into a general purpose finite element code:

- the input data should provide the diffuse rather than finite element connectivities,

- the $\mathbf{B}$ and $\mathbf{B}^{\mathrm{T}}$ matrices have to be replaced by their diffuse equivalents,

- the linear system solver has to deal with nonsymmetrical matrices.

\section{4}

\section{Boundary conditions}

In order to respect the Dirichlet boundary conditions we use an interpolating version of MLS with singular weight functions. The essential boundary conditions may thus be inserted directly into the global system in a usual way, for instance by zeroing the corresponding line, replacing the diagonal term by a unit value and inserting the known $\hat{u}$ value to the RHS.

The Neumann boundary conditions are imposed by a modification of the corresponding lines in $\mathbf{B}^{\mathrm{T}}$ in such a way to represent (16) rather than (14)

$\left.\mathbf{B T}=\left[\begin{array}{ccc} & \vdots & \\ M_{j, x} n_{x} & & M_{j, y} n_{y} \\ & \vdots & \\ & \vdots & \\ M_{j, x x} & & M_{j, y y} \\ & \vdots & \end{array}\right]\right\}$ Neumann-type conditions

Liszka et al. (1996) gives a method of enforcing the natural boundary conditions in GFDM by introducing supplementary "spectral" degrees of freedom on the boundary. This approach may be directly implemented in the double grid method.

\section{5}

\section{Numerical examples}

In the equations (14) and (16) we may choose the functions $f(x, y)$ and $g(x, y)$ in such a way that the exact solution of (14) corresponds to a given function $u_{e x}(x, y)$. It is obvious, considering that double grid is a second order approximation, that for all constant, linear or quadratic polynomial functions $u_{e x}$, a numerical solution corresponds to the exact one. Such tests are however useful for the validation of the computer program and they are satisfied with a very good accuracy (better than $10^{-10}$ ) for an arbitrary random mesh.

\section{1}

\section{Poisson's equation in 2D}

As the first example we present the Poisson's equation (14) in an unit square with the functions $f(x, y)$ and $g(x, y)$ such that

$u(x, y)=y^{2} \sin (k \pi x)$

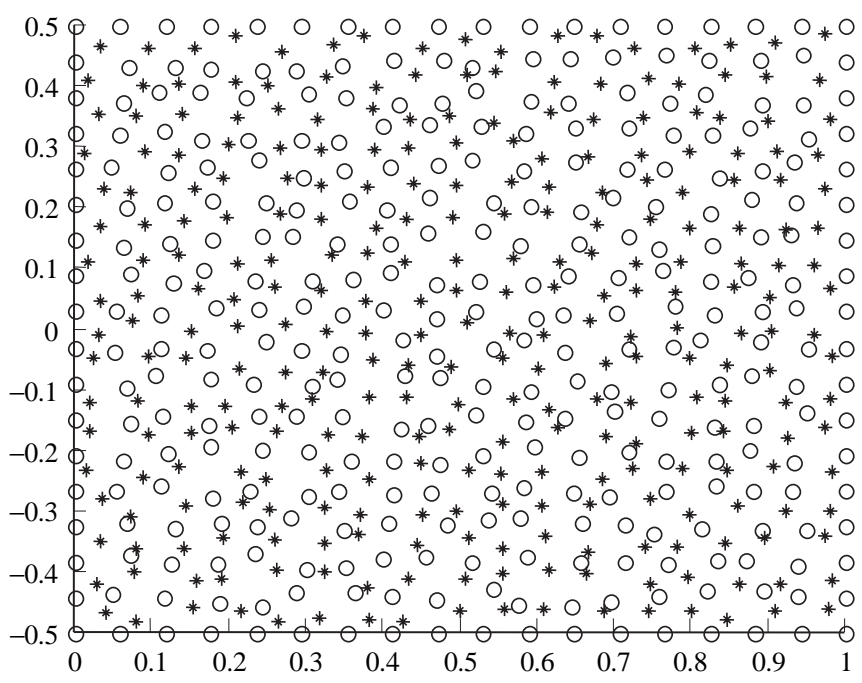

Fig. 5. A regular, randomly perturbed grid of nodes (stars) and of evaluation points (circles)

This example was studied using an irregular grid of nodes, the meshless methods being particularly interesting in such cases. In order to proceed to convergence tests we had to adopt a refinement strategy. We progressively refine a regular grid where the position of each point is perturbed by a random quantity of the order of magnitude of the grid step. The original regular grid step is $h$ and the perturbation coefficient is $\varepsilon$. In this way, the position of each internal point $i$ is modified for random values $\operatorname{rand}_{1}, \operatorname{rand}_{2} \in(0,1)$

$\Delta \mathbf{x}_{i}=\left(\varepsilon * h * \operatorname{rand}_{1}, \varepsilon * h * \operatorname{rand}_{2}\right)$

No comparison with a regular grid of nodes is possible because of the choice of the $L^{\infty}$ norm used to compute the $2 \mathrm{D}$ weight functions: the double grid approach is a second order method and it degenerates if the support nodes are located on a sphere in the sense of the chosen norm [Syczewski et al. (1981), Breitkopf et al. (1998)]. Figure 5 shows a double grid of $18 * 18$ nodes and $17 * 17$ evaluation points for $\varepsilon=0.5$.

The following figures show the rates of convergence obtained for different numbers of nodes connected to an evaluation point and vice-versa. Excepted the "under-connected" case of MLS based on four neighbors, the quadratic norm of the solution u converges at the rate of 2.15-2.82. The theoretical cubic rate of convergence of $u$ is confirmed when performing MLS approximation on irregular grid but this rate is not attained when solving equations. This fact may be related to the method of defining the domains of influence of collocation points. In fact, when the domains of influence are too small (the 4-points curves on Fig. 6 and on Fig. 7), the global matrix is badly conditioned as the domain is not sufficiently connected. On the other hand, when increasing the size of the elementary domains, the matrix becomes too "stiff" as it exhibits coupling terms for the nodes located far away from each other (the 7-points curve in Fig. 7). Further work is needed in order to improve the strategy for the definition of domains of influence. This 


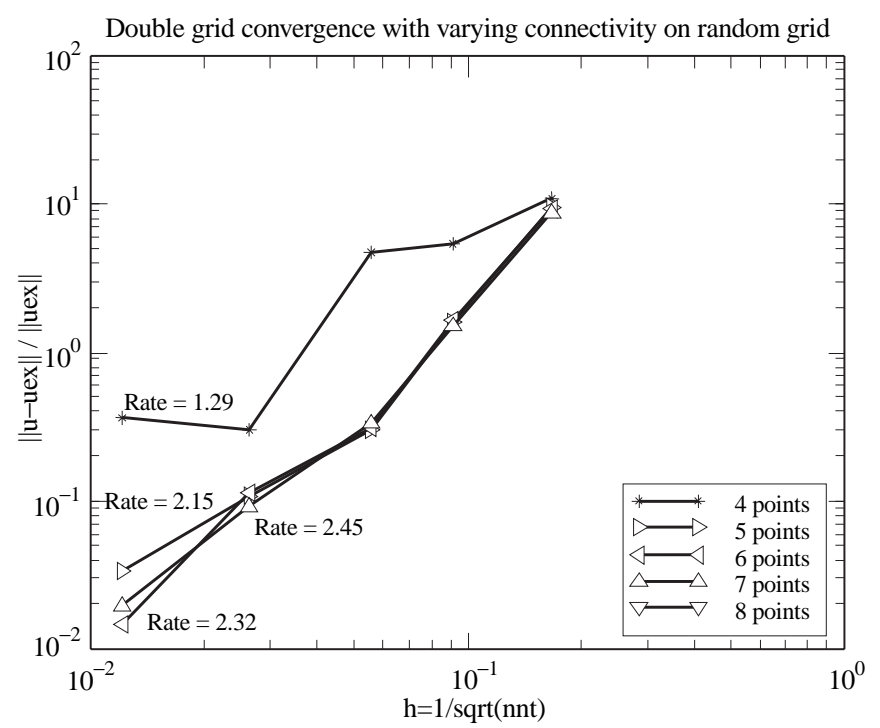

Fig. 6. Rates of convergence for different connectivities for a case with homogeneous Dirichlet boundary conditions

issue is crucial both for the collocation methods and for meshless weak formulations based on variational approaches [Orkisz (1998)].

The collocation methods are known to be sensitive to the way of imposing the boundary conditions of Neumann type [Liszka et al. (1996) and previous work by the same author]. We find this sensitivity when analyzing the Fig. 7 as compared to Fig. 6. This feature is more conspicuous in the case of equations of elasticity (next examples). In the present example the convergence rates are even slightly higher for the Neumann case but the corresponding solutions are less accurate.

\section{2}

\section{Cantilever beam}

In the second example we apply the double grid method for solving the equations of linear elasticity. This is illustrated here by a cantilever beam problem (Fig. 8).

The analytical solution given by Timoshenko et al. (1987) corresponds to a cubic field of displacements:

$$
\begin{aligned}
& u=\frac{-P y}{E I}\left(L x-\frac{x^{2}}{2}\right)+\frac{P}{I}\left(\frac{v}{E}-\frac{1}{G}\right) \frac{y^{3}}{6} \\
& v=\frac{P}{E I}\left(\left(L-\frac{x}{3}\right) \frac{x^{2}}{2}+v(L-x) \frac{y^{2}}{2}\right)+\frac{P H^{2} x}{8 G I} \\
& G=\frac{E}{2(1+v)} \\
& \sigma_{x}=\frac{-P y(L-x)}{I}, \quad \sigma_{y}=0, \quad \tau=\frac{P}{2 I}\left(\frac{H^{2}}{4}-y^{2}\right)
\end{aligned}
$$

The mesh is refined by the same technique as in the previous example. Figure 9 illustrates the case of a 25 -node and 36-evaluation points model of a square beam for $L=H=1$ loaded by an unit force $P$. The Young modulus $E=1$ and the Poisson's ratio is 0.3 . The Dirichlet boun

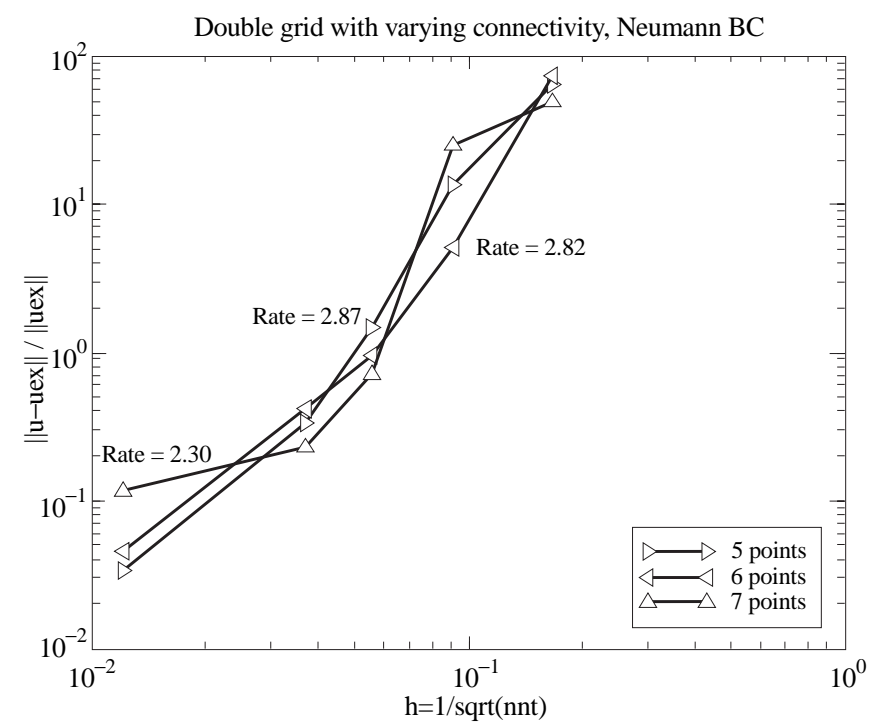

Fig. 7. Rates of convergence for the same example but with Dirichlet boundary conditions for $x=0$ and with Neumann boundary conditions on the rest of boundary

dary conditions are imposed for $x=0$ with the Neumann conditions on the rest of the boundary.

Figures 10 and 11 give the distribution of displacements and their derivatives along the axis of symmetry $y=0$ (the grid of points and of evaluation points are not symmet-

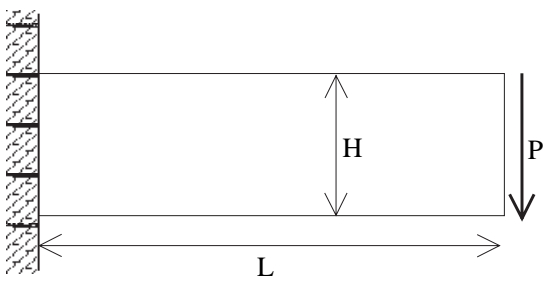

Fig. 8. The cantilever beam problem

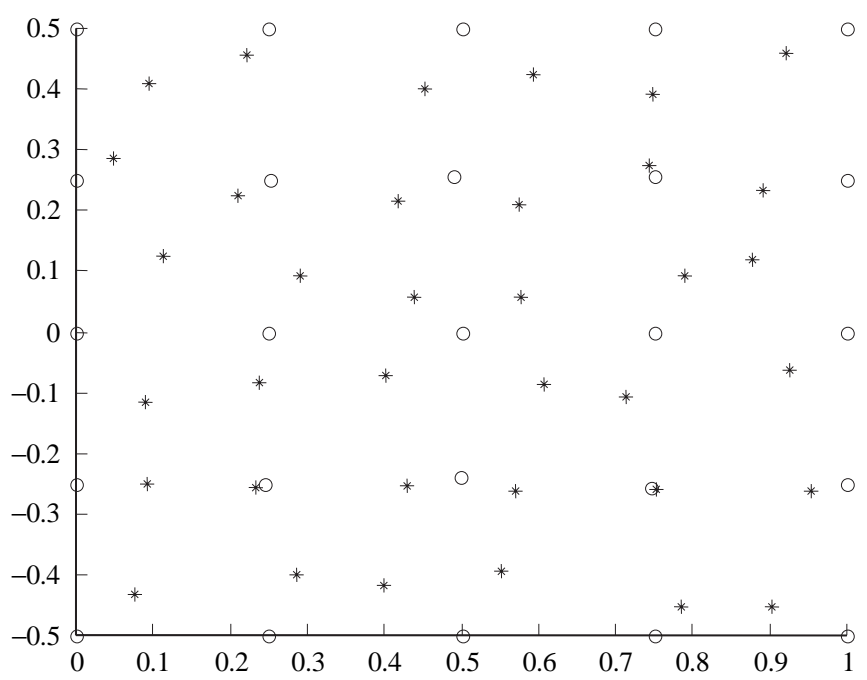

Fig. 9. Grid of nodes and of evaluation points obtained from a regular grid with step $h$ and perturbed by a random quantity $\varepsilon \in\langle 0,01 h\rangle$ for the nodes (circles) and $\varepsilon \in\langle 0,05 h\rangle$ for the evaluation points (stars) 


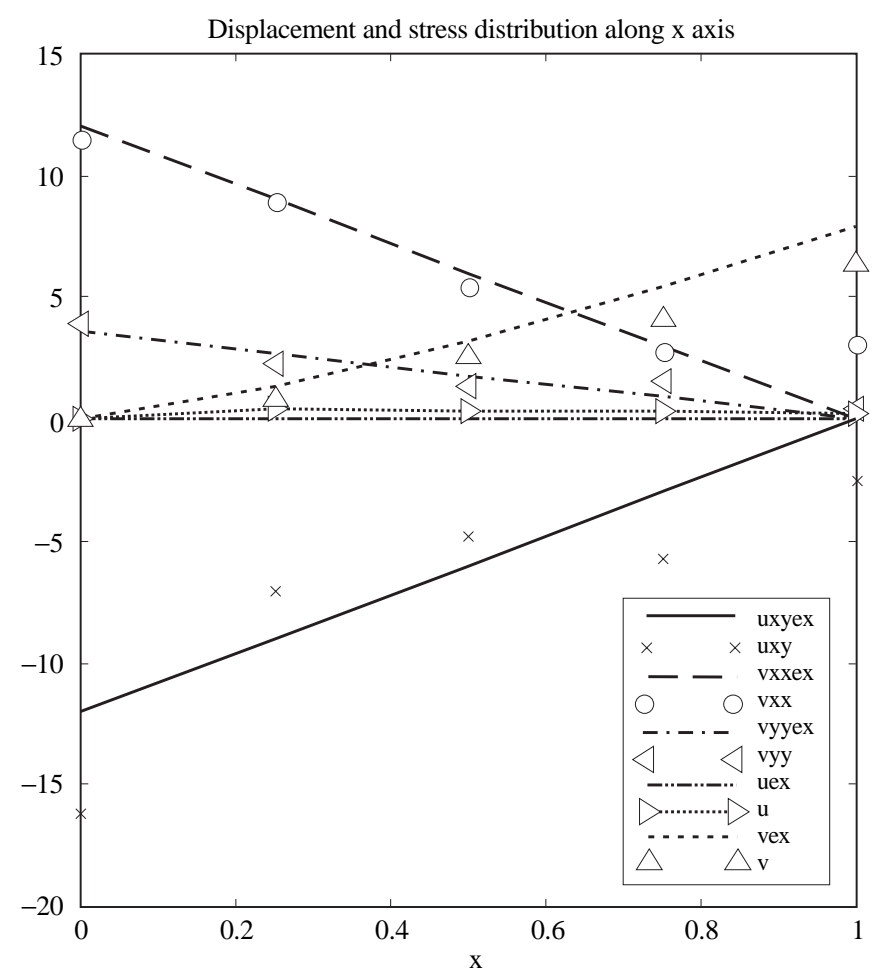

Fig. 10. 25 nodes and 36 evaluation points, $\left|u-u_{e x}\right| /\left|u_{e x}\right|=20 \%$

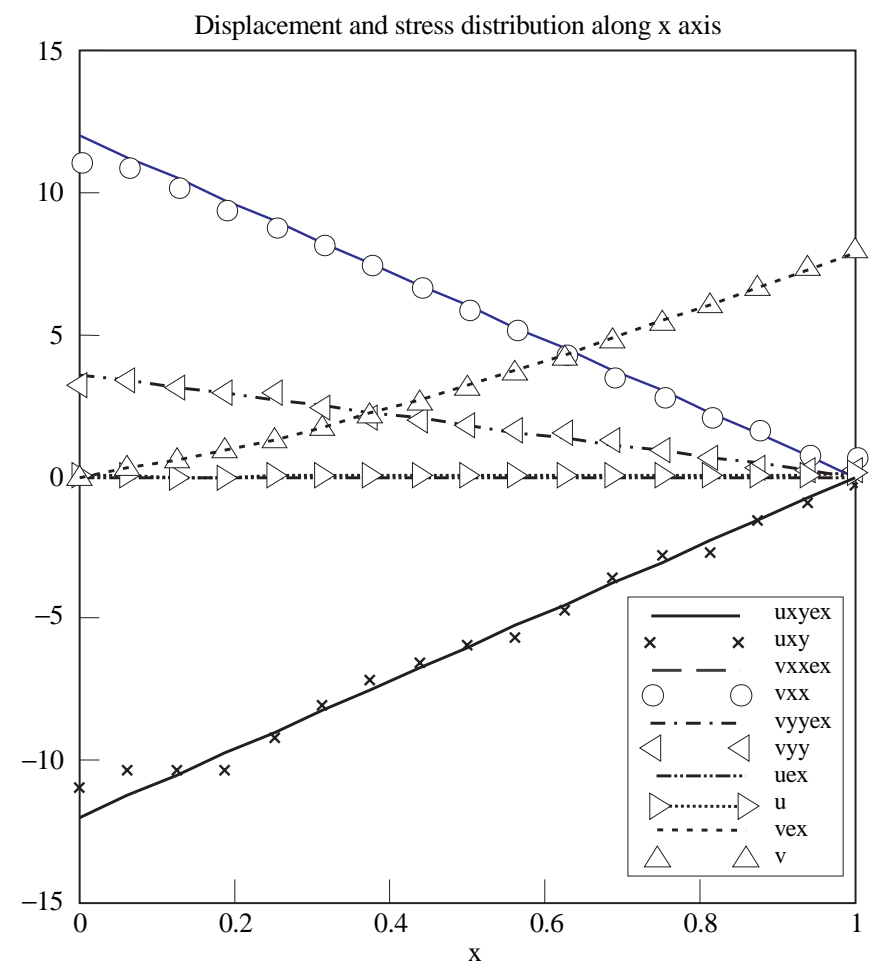

Fig. 11. 289 nodes and 324 evaluation points, $\left|u-u_{e x}\right| /$ $\left|u_{e x}\right|=2.35 \%$

rical). The plots are given for two cases: a coarse grid (Fig. 10) and a refined grid (Fig. 11). The quadratic norms of the following quantities are represented: $u, v,\left(\left(\partial^{2} u\right) /\left(\partial x^{2}\right)\right),\left(\left(\partial^{2} u\right) /(\partial x \partial y)\right),\left(\left(\partial^{2} u\right) /\left(\partial y^{2}\right)\right)$ and they are denoted on the graphs respectively: $u, v, u x x, u x y$, uyy. The continuous lines in the figures represent the exact quantities and the points correspond to the numerical solution.

One may observe good convergence of displacements. The second derivatives exhibit oscillations even for a refined mesh. This may explain the fact that the rate of convergence of displacements in this example is 1.79 and is significantly slower than that of the Poisson's case. The error on the second derivative approximation at the border nodes is inherent to the method as the pattern of points taken into account lies by definition on one side of the node. This lack of precision of the second derivatives may be observed in Fig. 11 at $x=0$ and $x=L$. It does not present any computational difficulties as the equations of equilibrium are replaced by the boundary conditions at these points.

\section{Conclusion}

The double grid approach is proposed as an alternative to the GFDM. The obtained rates of convergence are similar to those observed by Liszka et al. (1996) in GFDM. The main difference between the methods lies in the strategy of choosing the nodal connectivities. The selection of "stars" or "stencils" in GFDM results in an arbitrary selection of points taken into account when evaluating the approximating coefficients. Therefore the resulting GFDM shape functions are not continuous. The reason not for taking into account all the nodes belonging to a domain of influence are twofold: the numerical conditioning and the density of the resulting global matrix. In the double grid method we limit the required connectivity by splitting the second order operator into the two first order operators. The resulting domains of influence being smaller, the sparsity of the global matrix is enforced. Excepting the well known degenerate patterns we did not encounter conditioning problems.

On the other hand, the double grid leads to an interesting structure of the computer code, similar to that of the finite elements. In some sense, the operation $\mathbf{B T} * \mathbf{D} * \mathbf{B}$ resembles to that of integrating the finite element stiffness matrix with the integration of the $\mathbf{B}^{\mathrm{T}} * \mathbf{D} * \mathbf{B}$ terms. It is therefore straightforward to include the double grid and a meshless variational method (Diffuse Elements or EFG) in the same program.

The double grid method is however still in an early phase of its development and further work is needed in order to decrease the oscillations of the second derivative. An interesting study would consist in an adaptation of the method to highly irregular grids with strong variations of the density of nodes resulting for instance from autoadaptive computations.

\section{References}

Belytschko T, Lu YY, Gu L (1994) Element-free Galerkin Methods. Int. J. Num. Meth. Eng. 37:229-256

Breitkopf P, Touzot G, Villon P (1998) Consistency approach and diffuse derivation in element free methods based on moving least squares approximation. Comp. Assist. Mech. Eng. Sci. 5:479-501 
Liszka T (1984) An Interpolation method for an irregular net of nodes. Int. J. Num. Meth. Eng. 20:1599-1612

Liszka T, Orkisz J (1980) The finite difference method at arbitrary irregular grids and its application in applied mechanics. Comp. Struct. 11:83-95

Liszka T, Orkisz J (1983) Solution of nonlinear problems of mechanics by the finite difference method at arbitrary meshes. Comp. Mech. 5:117-130

Liszka TJ, Duarte CAM, Tworzydlo WW (1996) hp-Meshless cloud method. Comput. Meth. Appl. Mech. Eng. 139
Nayroles B, Touzot G, Villon P (1992) Generalizing the finite element method: diffuse approximation and diffuse elements. Comput. Mech. 10:307-318

Orkisz J (1998) Meshless finite difference method. I. Basic approach, II. Adaptive approach, IACM-Fourth World Congress on computational mechanics, Buenos Aires

Syczewski M, Tribillo R (1981) Singularities of sets used in the mesh method. Comp. Struct. 14(5-6):509-511

Timoshenko SP, Goodier JN (1987) Theory of elasticity, 3rd edition, McGraw-Hill, New York 